\title{
How to care for a patient after death in the community
}

Greenway K, Johnson P (2016) How to care for a patient after death in the community.

Nursing Standard. 30, 37, 34-36. Date of submission: February 12 2016; date of acceptance: February 262016.

\section{Rationale and key points}

This article provides nurses with information about how to care for a patient after death, and support their family and loved ones in the community setting.

- Care after death involves supporting the family and significant others, and providing personal care to the patient.

- It is important to ensure privacy, dignity and respect of the deceased and to recognise this is a sensitive and difficult time for families.

- Staff undertaking care after death should be offered support.

- Local and national guidelines should be followed.

\section{Reflective activity}

Clinical skills articles can help update your practice and ensure it remains evidence based. Apply this article to your practice, reflect on and write a short account of:

1. How reading this article will change your practice.

2. How this article could help you to consider the support mechanisms in place for the provision of care after death in the community setting. Subscribers can upload their reflective accounts at: rcni.com/portfolio.

\section{Authors}

Kathleen Greenway Senior lecturer in adult nursing.

Paula Johnson Senior lecturer in adult nursing.

Both at Oxford Brookes University, Oxford, England.

Correspondence to: kgreenway@brookes.ac.uk @kgreenwaynurse

\section{Keywords}

care after death, clinical procedures, clinical skills, communication, community setting, family support, personal care

\section{Contributing to the clinical skills series}

To write a clinical skills article, please email How.to@rcni.com with a synopsis of your idea.

\section{Review}

All articles are subject to external double-blind peer review and checked for plagiarism using automated software.

\section{Online}

This 'How to' guide is available at: rcni.com/how-to. For related articles search the website using the keywords above.

\section{Preparation and equipment}

- It is acknowledged that nurses may not always be involved in care after death in the community setting. Funeral directors or other healthcare professionals may undertake this role. For ease of reading, the term 'nurse' will be used in this article.

- The nurse should identify and attend to patient and family preferences and cultural beliefs when performing care after death. They should be aware of any advance care planning documentation for the patient to fulfil their wishes.

- The nurse should be aware of the circumstances in which a death would be reported to a coroner, for example if the death is unexplained. If a death appears suspicious, the police must be contacted immediately. In this case, the patient's body should not be washed and mouth care should not be performed. All devices and prosthetics must remain in place.

- The nurse should ensure the necessary equipment is available, including:

- Occlusive tape.

- Gloves and an apron.

- Clean sheets.

- Towel.

- Slide sheet or hoist sling.

- Toothbrush or sponge sticks.

- Absorbent pads or dressings or continence pad or pants.

- Clinical and domestic waste bags.

- Bowl.

- Spigot(s).

- Verification of death forms.

\section{Procedure}

Those undertaking personal care for the deceased should ensure their privacy, dignity and respect at all times.

1. Record the time of death and time of verification of death in the patient documentation and on the verification of death form. This can be completed by a nurse with this extended role or the patient's GP, while 
adhering to local and national guidelines (Hospice UK and National Nurse Consultant Group (Palliative Care) (NNCG) 2015).

2. Contact the next of kin and significant others to inform them of the patient's death if they are not present. Be aware that receiving such news may be distressing.

3. Acknowledge and discuss any preferences that the patient or family have noted that relate to the care after death procedure. This may include cultural or religious preferences or requirements.

4. Inform the GP of the patient's death.

5. Contact the funeral director chosen by the family to arrange removal of the deceased.

6. Wash your hands and put on appropriate personal protective equipment, including gloves and an apron, before undertaking any personal care, as per local policy.

7. Position the patient on their back using the appropriate equipment, as per local manual handling policy. It is important to straighten the limbs before rigor mortis begins.

8. Close the patient's eyes by applying light pressure for 30 seconds.

9. Wash the patient, using a bowl, water, toiletries and towel. The patient's toiletries can be used if they are available. Ensure that the patient's privacy and dignity are maintained. Family members may wish to be involved with this aspect of care, and it is important that this is confirmed and they are aware of what to expect. Family involvement may not be appropriate if there are infection control issues.

10. Perform oral hygiene using a toothbrush or sponge sticks. This may include cleaning and reinserting the patient's dentures. If you are unable to reinsert the dentures, they should be sent to the funeral director with the body.

11. Shaving a recently deceased person is not recommended, since the skin is still warm and bruising and marking may appear days later (Hospice UK and NNCG 2015). The funeral director can shave the patient at a later date, if the family requests this.

12. Drainage and intravenous catheters can be removed. If the death is being referred to a coroner, or to the procurator fiscal in Scotland to investigate the cause, all devices and prosthetics must remain in place.

13. Use absorbent dressings and occlusive tape to prevent any leakage from wounds or drainage sites, to protect staff from any exposure to body fluids.

14. The body continues to excrete fluids after death. Apply or position pads to absorb any leakage and prevent soiling. Spigots should be used to prevent any drainage devices from leaking.

15. Place a clean sheet beneath the deceased, using appropriate manual handling equipment, such as a slide sheet or hoist sling, and adhering to local manual handling policy.

16. Discuss with the family if any jewellery on the patient is to be removed or left in place. If any jewellery remains on the body, this should be recorded in the funeral director's documentation. If the patient is not in their home, follow local policy regarding the patient's property.

17. Dress the deceased in clean personal clothing. You should take into account the family's preferences and adhere to local policy, especially if the deceased presents an infection risk to the funeral director.

18. In preparation for removal of the body by the funeral director, place a clean sheet over the deceased. This may include covering the deceased's face in accordance with the family's wishes. If there is a high risk of infection, a body bag provided by the funeral director should be used (Health and Safety Executive 2005).

19. Dispose of used equipment according to local policy. Use clinical waste bags for the disposal of any waste considered infectious or hazardous. To reduce the risk of cross-infection, decontaminate your hands by washing or using alcohol hand gel (Loveday et al 2014).

20. Provide the family and significant others with an opportunity to discuss or reflect on the death and care provided to the deceased.

21. Record all actions carried out in the medical and nursing documentation.

\section{Evidence base}

Following national recommendations, the term 'last offices' has been replaced by the more inclusive term 'care after death' (Hospice UK and NNCG 2015). Personal care after death refers to physical care of the patient's body. Nurses should view care after death as a continuation of the person-centred care they provided while the patient was alive. It is important that the care which nurses provide to the patient after death conveys respect for the patient and their family, and fulfils any religious or cultural obligations or beliefs (Leadership Alliance for the Care of Dying People 2014, Martin and Bristowe 2015).

Healthcare professionals develop unique relationships with patients and may also grieve for the deceased. It is important to recognise distress in colleagues following care after death and provide appropriate support. Positive experiences in relation to care after death, alongside additional support and education, can enable staff to develop coping strategies in the future (Gerow et al 2010). 
The nurse should discuss the patient's personal care after death with the family and significant others from the outset, and reflect on the non-verbal cues exhibited by them. It is important that the family and loved ones do not feel pressured into being involved in personal care after death. Although these individuals may have been present at the patient's bedside before and after death, the nurse should not assume that they will choose to be involved in personal care after death (Martin and Bristowe 2015) NS

Disclaimer: please note that information provided by Nursing Standard is not sufficient to make the reader competent to perform the task. All clinical skills should be formally assessed at the bedside by a nurse educator or mentor. It is the nurse's responsibility to ensure their practice remains up to date and reflects the latest evidence.

\section{USEFUL RESOURCES}

- National Institute for Health and Care Excellence (2011) End of Life Care for Adults. Quality Standard No. 13. www.nice.org.uk/guidance/qs13

- National Institute for Health and Care Excellence (2015) Care of Dying Adults in the Last Days of Life. Guideline No. 31. www.nice.org.uk/guidance/ng31 (Last accessed: April 6 2016.)

\section{References}

Gerow L, Conejo P, Alonzo A, Davis N, Rodgers S, Domian EW (2010) Creating a curtain of protection: nurses' experiences of grief following patient death. Journal of Nursing Scholarship. $42,2,122-129$.

Health and Safety Executive (2005) Controlling the Risks of Infection at Work from Human Remains: A Guide for Those Involved in Funeral Services (Including Embalmers) and Those Involved in Exhumation. www.hse.gov.uk/pubns/web01.pdf (Last accessed: April 6 2016.)

Hospice UK and National Nurse Consultant Group (Palliative Care) (2015) Care After Death: Guidance for Staff Responsible for Care After Death. tinyurl.com/q62gmpd (Last accessed: April 6 2016.)

Leadership Alliance for the Care of Dying People (2014) One Chance To Get It Right: Improving People's Experience of Care in the Last Few Days and Hours of Life. www.gov.uk/government/ uploads/system/uploads/attachment_data/file/323188/0ne_ chance_to_get_it_right.pdf (Last accessed: April 6 2016.)

Loveday HP, Wilson JA, Pratt RJ et al (2014) epic3: national evidence-based guidelines for preventing healthcare-associated infections in NHS hospitals in England. Journal of Hospital Infection. 86, Suppl 1, S1-S70.

Martin S, Bristowe K (2015) Last offices: nurses' experiences of the process and their views about involving significant others. International Journal of Palliative Nursing. 21, 4, 173-178.

\title{
NURSINGSTANDARD
}
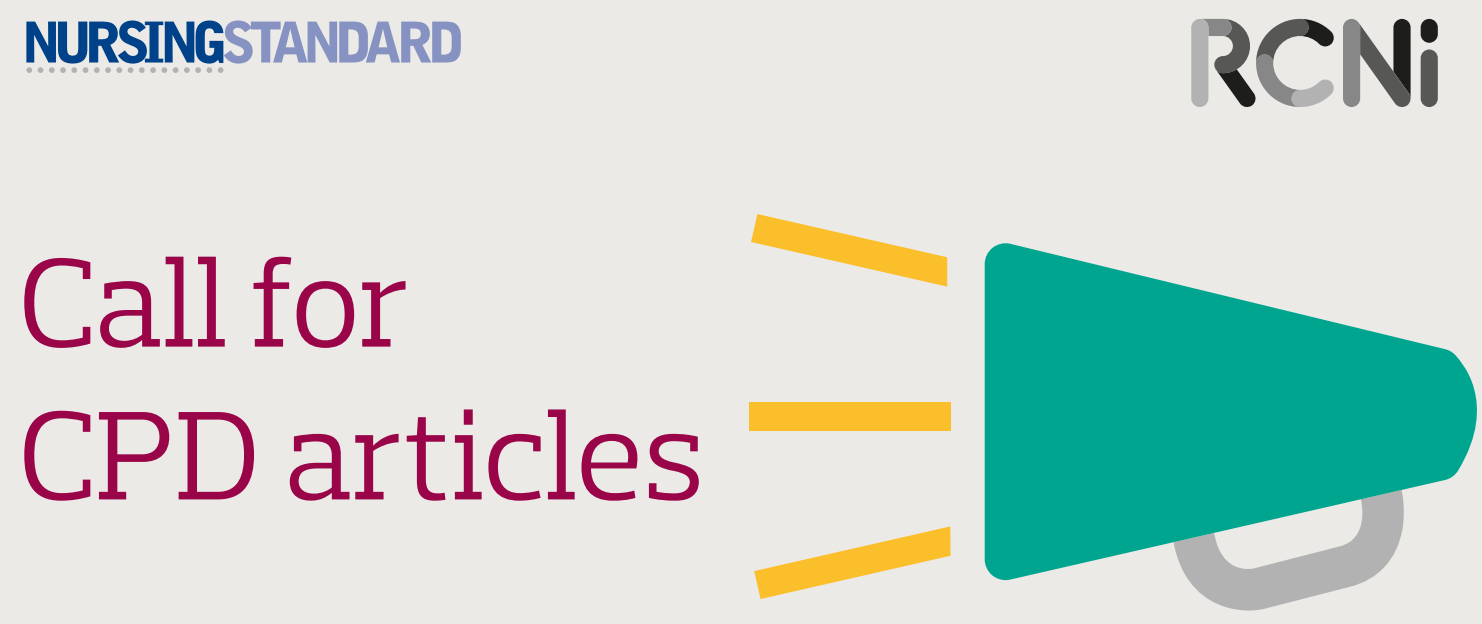

\author{
Nursing Standard is welcoming submissions from \\ experienced or new authors on a variety of subjects
}

